\title{
Under-Approximating Cut Sets for Reachability in Large Scale Automata Networks
}

\author{
Loïc Paulevé ${ }^{1}$, Geoffroy Andrieux ${ }^{2}$, and Heinz Koeppl ${ }^{1,3}$ \\ 1 ETH Zürich, Switzerland \\ 2 IRISA Rennes, France \\ 3 IBM Research - Zurich, Rueschlikon, Switzerland
}

\begin{abstract}
In the scope of discrete finite-state models of interacting components, we present a novel algorithm for identifying sets of local states of components whose activity is necessary for the reachability of a given local state. If all the local states from such a set are disabled in the model, the concerned reachability is impossible.

Those sets are referred to as cut sets and are computed from a particular abstract causality structure, so-called Graph of Local Causality, inspired from previous work and generalised here to finite automata networks. The extracted sets of local states form an under-approximation of the complete minimal cut sets of the dynamics: there may exist smaller or additional cut sets for the given reachability.

Applied to qualitative models of biological systems, such cut sets provide potential therapeutic targets that are proven to prevent molecules of interest to become active, up to the correctness of the model. Our new method makes tractable the formal analysis of very large scale networks, as illustrated by the computation of cut sets within a Boolean model of biological pathways interactions gathering more than 9000 components.
\end{abstract}

\section{Introduction}

With the aim of understanding and, ultimately, controlling physical systems, one generally constructs dynamical models of the known interactions between the components of the system. Because parts of those physical processes are ignored or still unknown, dynamics of such models aim at over-approximating the real system dynamics: any (observed) behaviour of the real system has to have a matching behaviour in the abstract model, the converse being potentially false. In such a setting, a valuable contribution of formal methods on abstract models of physical systems resides in the ability to prove the impossibility of particular behaviours.

Given a discrete finite-state model of interacting components, such as an automata network, we address here the computation of sets of local states of components that are necessary for reaching a local state of interest from a partially determined initial global state. Those sets are referred to as cut sets. Informally, each path leading to the reachability of interest has to involve, at one point, at least one local state of a cut set. Hence, disabling in the model all the local states referenced in one cut set should prevent the occurrence of the concerned reachability from delimited initial states. This is illustrated by Fig. 1. 


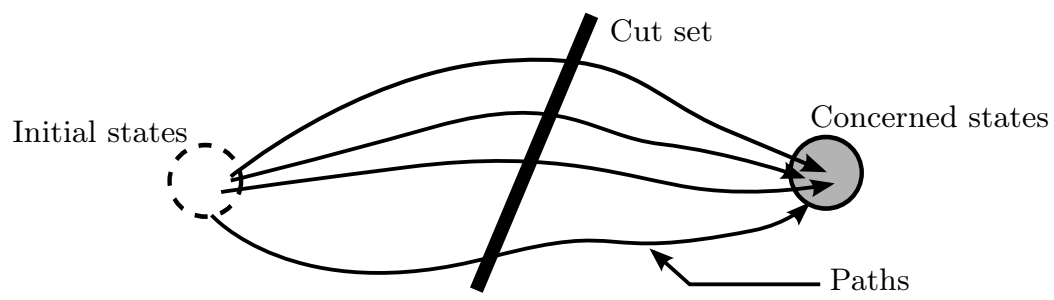

Fig. 1. A cut set is composed of local states that are involved in all paths from delimited initial states to concerned states. Disabling all the local states from such a cut set necessarily breaks the concerned reachability in the model.

Applied to a model of a biological system where the reachability of interest is known to occur, such cut sets provide potential coupled therapeutic targets to control the activity of a particular molecule (for instance using gene knock-in/out). The contrary implies that the abstract model is not an overapproximation of the concrete system.

Contribution. In this paper, we present a new algorithm to extract sets of local states that are necessary to achieve the concerned reachability within a finite automata network. Those sets are referred to as cut sets, and we limit ourselves to $N$-sets, i.e. having a maximum cardinality of $N$.

The finite automata networks we are considering are closely related to 1safe Petri nets [1] having mutually exclusive places. They subsume Boolean and discrete networks 92417/2], synchronous or asynchronous, that are widely used for the qualitative modelling of biological interaction networks.

A naive, but complete, algorithm could enumerate all potential candidate $N$-sets, disable each of them in the model, and then perform model-checking to verify if the targeted reachability is still verified. If not, the candidate $N$ set is a cut set. This would roughly leads to $m^{N}$ tests, where $m$ is the total number of local states in the automata network. Considering that the modelchecking within automata networks is PSPACE-complete [5], this makes such an approach intractable on large networks.

The proposed algorithm aims at being tractable on systems composed of a very large number of interacting components, but each of them having a small number of local states. Our method principally overcomes two challenges: prevent a complete enumeration of candidate $N$-sets; and prevent the use of model-checking to verify if disabling a set of local states break the concerned reachability. It inherently handles partially-determined initial states: the resulting cut $N$-set of local states are proven to be necessary for the reachability of the local state of interest from any of the supplied global initial states.

The computation of the cut $N$-sets takes advantage of an abstraction of the formal model which highlights some steps that are necessary to occur prior to the verification of a given reachability property. This results in a causality structure called a Graph of Local Causality (GLC), which is inspired by [16], and that we generalise here to automata networks. Such a GLC has a size polynomial with 
the total number of local states in the automata network, and exponential with the number of local states within one automata. Given a GLC, our algorithm propagates and combines the cut $N$-sets of the local states referenced in this graph by computing unions or products, depending on the disjunctive or conjunctive relations between the necessary conditions for their reachability. The algorithm is proven to converge in the presence of dependence cycles.

In order to demonstrate the scalability of our approach, we have computed cut $N$-sets within a very large Boolean model of a biological network relating more than 9000 components. Despite the highly combinatorial dynamics, a prototype implementation manages to compute up to the cut 5-sets within a few minutes. To our knowledge, this is the first time such a formal dynamical analysis has been performed on such a large dynamical model of biological system.

Related work and limitations. Cut sets are commonly defined upon graphs as set of edges or vertices which, if removed, disconnect a given pair of nodes [21. For our purpose, this approach could be directly applied to the global transition graph to identify local states or transitions for which the removal would disconnect initial states from the targeted states. However, the combinatorial explosion of the state space would make it intractable for large interacting systems.

The aim of the presented method is somehow similar to the generation of minimal cut sets in fault trees 1322 used for reliability analysis, as the structure representing reachability causality contains both and and or connectors. However, the major difference is that we are here dealing with cyclic directed graphs which prevents the above mentioned methods to be straightforwardly applied.

Klamt et al. have developed a complete method for identifying minimal cut sets (also called intervention sets) dedicated to biochemical reactions networks, hence involving cycling dependencies [10]. This method has been later generalised to Boolean models of signalling networks [19]. Those algorithms are mainly based on the enumeration of possible candidates, with techniques to reduce the search space, for instance by exploiting symmetry of dynamics. Whereas intervention sets of [1019] can contain either local states or reactions, our cut sets are only composed of local states.

Our method follows a different approach than [10 19 by not relying on candidate enumeration but computing the cut sets directly on an abstract structure derived statically from the model, which should make tractable the analysis of very large networks. The comparison with [19] is detailed in Subsect. 4.1.

In addition, our method is generic to any automata network, but relies on an abstract interpretation of dynamics which leads to under-approximating the cut sets for reachability: by ignoring certain dynamical constraints, the analysis can miss several cut sets and output cut sets that are not minimal for the concrete model. Finally, although we focus on finding the cut sets for the reachability of only one local state, our algorithm computes the cut sets for the (independent) reachability of all local states referenced in the GLC. 
Outline. Sect. 2 introduces a generic characterisation of the Graph of Local Causality with respect to automata networks; Sect. 3 states and sketches the proof of the algorithm for extracting a subset of $N$-sets of local states necessary for the reachability of a given local state. Sect. 4 discusses the application to systems biology by comparing with the related work and applying our new method to a very large scale model of biological interactions. Finally, Sect. 5 ] discusses the results presented and some of their possible extensions.

Notations. $\wedge$ and $\vee$ are the usual logical and and or connectors. $[1 ; n]=$ $\{1, \cdots, n\}$. Given a finite set $A, \# A$ is the cardinality of $A ; \wp(A)$ is the power set of $A ; \wp^{\leq} \leq N(A)$ is the set of all subsets of $A$ with cardinality at most $N$. Given sets $A^{1}, \ldots, A^{n}, \bigcup_{i \in[1 ; n]} A^{i}$ is the union of those sets, with the empty union $\bigcup_{\emptyset} \triangleq \emptyset$; and $A^{1} \times \cdots \times A^{n}$ is the usual Cartesian product. Given sets of sets $B^{1}, \ldots, B^{n} \in \wp(\wp(A)), \widetilde{\prod}_{i \in[1 ; n]} B^{i} \triangleq B^{1} \tilde{\times} \cdots \tilde{\times} B^{n} \in \wp(\wp(A))$ is the sets of sets product where $\left\{e_{1}, \ldots, e_{n}\right\} \tilde{\times}\left\{e_{1}^{\prime}, \ldots, e_{m}^{\prime}\right\} \triangleq\left\{e_{i} \cup e_{j}^{\prime} \mid i \in[1 ; n] \wedge j \in[1 ; m]\right\}$. In particular $\forall(i, j) \in[1 ; n] \times[1 ; m], B^{i} \tilde{\times} B^{j}=B^{j} \tilde{\times} B^{i}$ and $\emptyset \tilde{\times} B^{i}=\emptyset$. The empty sets of sets product $\widetilde{\prod}_{\emptyset} \triangleq\{\emptyset\}$. If $M: A \mapsto B$ is a mapping from elements in $A$ to elements in $B, M(a)$ is the value in $B$ mapped to $a \in A ; M\{a \mapsto b\}$ is the mapping $M$ where $a \in A$ now maps to $b \in B$.

\section{Graph of Local Causality}

We first give basic definitions of automata networks, local state disabling, context and local state reachability; then we define the local causality of an objective (local reachability), and the Graph of Local Causality. A simple example is given at the end of the section.

\subsection{Finite Automata Networks}

We consider a network of automata $(\Sigma, S, \mathcal{L}, T)$ which relates a finite number of interacting finite state automata $\Sigma$ (Def. 1). The global state of the system is the gathering of the local state of composing automata. A transition can occur if and only if all the local states sharing a common transition label $\ell \in L$ are present in the global state $s \in S$ of the system. Such networks characterize a class of 1-safe Petri Nets [1] having groups of mutually exclusive places, acting as the automata. They allow the modelling of Boolean networks and their discrete generalisation, having either synchronous or asynchronous transitions.

Definition 1 (Automata Network $(\Sigma, S, \mathcal{L}, T)$ ). An automata network is defined by a tuple $(\Sigma, S, \mathcal{L}, T)$ where

$-\Sigma=\{a, b, \ldots, z\}$ is the finite set of automata identifiers;

- For any $a \in \Sigma, S(a)=\left[1 ; k_{a}\right]$ is the finite set of local states of automaton $a$; $S=\prod_{a \in \Sigma}\left[1 ; k_{a}\right]$ is the finite set of global states.

$-\mathcal{L}=\left\{\ell_{1}, \ldots, \ell_{m}\right\}$ is the finite set of transition labels; 
- $T=\left\{a \mapsto T_{a} \mid a \in \Sigma\right\}$, where $\forall a \in \Sigma, T_{a} \subset\left[1 ; k_{a}\right] \times \mathcal{L} \times\left[1 ; k_{a}\right]$, is the mapping from automata to their finite set of local transitions.

We note $i \stackrel{\ell}{\rightarrow} j \in T(a) \stackrel{\Delta}{\Leftrightarrow}(i, \ell, j) \in T_{a}$ and $a_{i} \stackrel{\ell}{\rightarrow} a_{j} \in T \stackrel{\Delta}{\Leftrightarrow} i \stackrel{\ell}{\rightarrow} j \in T(a)$.

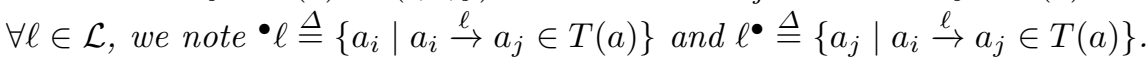

The set of local states is defined as $\mathbf{L S} \triangleq\left\{a_{i} \mid a \in \Sigma \wedge i \in\left[1 ; k_{a}\right]\right\}$.

The global transition relation $\rightarrow \subset S \times S$ is defined as:

$$
\begin{gathered}
s \rightarrow s^{\prime} \stackrel{\Delta}{\Leftrightarrow} \exists \ell \in \mathcal{L}: \forall a_{i} \in \bullet \ell, s(a)=a_{i} \wedge \forall a_{j} \in \ell^{\bullet}, s^{\prime}(a)=a_{j} \\
\wedge \forall b \in \Sigma, S(b) \cap \bullet \ell=\emptyset \Rightarrow s(b)=s^{\prime}(b) .
\end{gathered}
$$

Given an automata network $\mathcal{S} y s=(\Sigma, S, \mathcal{L}, T)$ and a subset of its local states $l s \subseteq \mathbf{L S}, \mathcal{S} y s \ominus l s$ refers to the system where all the local states $l s$ have been disabled, i.e. they can not be involved in any transition (Def. 2).

Definition 2 (Local states disabling). Given $\mathcal{S} y s=(\Sigma, S, \mathcal{L}, T)$ and $l s \in$ $\wp(\mathbf{L S}), \mathcal{S} y s \ominus l s \triangleq\left(\Sigma, S, \mathcal{L}^{\prime}, T^{\prime}\right)$ where $\mathcal{L}^{\prime}=\{\ell \in \mathcal{L} \mid$ ls $\cap \bullet \ell=\emptyset\}$ and $T^{\prime}=$ $\left\{a_{i} \stackrel{\ell}{\rightarrow} a_{j} \in T \mid \ell \in \mathcal{L}^{\prime}\right\}$.

From a set of acceptable initial states delimited by a context $\varsigma$ (Def. [3), we say a given local state $a_{j} \in \mathbf{L S}$ is reachable if and only if there exists a finite number of transitions in $\mathcal{S} y s$ leading to a global state where $a_{j}$ is present (Def. 4).

Definition 3 (Context $\varsigma$ ). Given a network $(\Sigma, S, \mathcal{L}, T)$, a context $\varsigma$ is a mapping from each automaton $a \in \Sigma$ to a non-empty subset of its local states: $\forall a \in \Sigma, \varsigma(a) \in \wp(S(a)) \wedge \varsigma(a) \neq \emptyset$.

Definition 4 (Local state reachability). Given a network $(\Sigma, S, \mathcal{L}, T)$ and a context $\varsigma$, the local state $a_{j} \in \mathbf{L S}$ is reachable from $\varsigma$ if and only if $\exists s_{0}, \ldots, s_{m} \in$ $S$ such that $\forall a \in \Sigma, s_{0}(a) \in \varsigma(a)$, and $s_{0} \rightarrow \cdots \rightarrow s_{m}$, and $s_{m}(a)=j$.

\subsection{Local Causality}

Locally reasoning within one automaton $a$, the global reachability of $a_{j}$ from $\varsigma$ can be expressed as the reachability of $a_{j}$ from a local state $a_{i} \in \varsigma(a)$. This local reachability specification is referred to as an objective noted $a_{i} \rightarrow^{*} a_{j}$ (Def. [5).

Definition 5 (Objective). Given a network $(\Sigma, S, \mathcal{L}, T)$, the reachability of local state $a_{j}$ from $a_{i}$ is called an objective and is denoted $a_{i} \rightarrow^{*} a_{j}$. The set of all objectives is referred to as $\mathbf{O b j} \triangleq\left\{a_{i} \rightarrow^{*} a_{j} \mid\left(a_{i}, a_{j}\right) \in \mathbf{L S} \times \mathbf{L S}\right\}$.

Given an objective $P=a_{i} \rightarrow^{*} a_{j} \in \mathbf{O b j}$, we define sol $(P)$ the local causality of $P$ (Def. 6): each $l s \in \operatorname{sol}(P)$ is a set of local states that may be involved for the reachability of $a_{j}$ from $a_{i} ; l s$ is referred to as a (local) solution for $P$. $\operatorname{sol}(P)$ is sound as soon as the disabling of at least one local state in each solution makes the reachability of $a_{j}$ impossible from any global state containing $a_{i}$ (Property 1 ). It implies that if $\operatorname{sol}(P)=\left\{\left\{a_{i}\right\} \cup l s^{1}, \ldots, l s^{m}\right\}$ is sound, $\operatorname{sol}^{\prime}(P)=\left\{l s^{1}, \ldots, l s^{m}\right\}$ is also sound. $\operatorname{sol}\left(a_{i} \rightarrow^{*} a_{j}\right)=\emptyset$ implies that $a_{j}$ can never be reached from $a_{i}$, and $\forall a_{i} \in \mathbf{L S}, \operatorname{sol}\left(a_{i} \rightarrow^{*} a_{i}\right) \triangleq\{\emptyset\}$. 
Definition 6. sol : Obj $\mapsto \wp(\wp(\mathbf{L S}))$ is a mapping from objectives to sets of sets of local states such that $\forall P \in \mathbf{O b j}, \forall l s \in \operatorname{sol}(P), \nexists l s^{\prime} \in \operatorname{sol}(P), l s \neq l s^{\prime}$ such that $l s^{\prime} \subset l s$. The set of these mappings is noted $\mathbf{S o l} \triangleq\{\langle P, l s\rangle \mid l s \in \operatorname{sol}(P)\}$.

Property 1 (sol soundness). $\operatorname{sol}\left(a_{i} \rightarrow^{*} a_{j}\right)=\left\{l s^{1}, \ldots, l s^{n}\right\}$ is a sound set of solutions for the network $\mathcal{S} y s=(\Sigma, S, \mathcal{L}, T)$ if and only if $\forall k l s \in \widetilde{\prod}_{i \in[1 ; n]}\left\{l s^{i}\right\}, a_{j}$ is not reachable in $\mathcal{S} y s \ominus k l s$ from any state $s \in S$ such that $s(a)=i$.

In the rest of this paper we assume that Property 1 is satisfied, and consider sol computation out of the scope of this paper.

Nevertheless, we briefly describe a construction of a sound sol $\left(a_{i} \rightarrow^{*} a_{j}\right)$ for an automata network $(\Sigma, S, \mathcal{L}, T)$; an example is given at the end of this section. This construction generalises the computation of GLC from the Process Hitting framework, a restriction of network of automata depicted in [16]. For each acyclic sequence $a_{i} \stackrel{\ell_{1}}{\longrightarrow} \ldots \stackrel{\ell_{m}}{\longrightarrow} a_{j}$ of local transitions in $T(a)$, and by defining ext ${ }_{a}(\ell) \triangleq$ $\left\{b_{j} \in \mathbf{L S} \mid b_{j} \stackrel{\ell}{\rightarrow} b_{k} \in T, b \neq a\right\}$, we set $l s \in \widetilde{\prod}_{\ell \in\left\{\ell_{1}, \ldots, \ell_{m} \mid \operatorname{ext}_{a}(\ell) \neq \emptyset\right\}}\left\{\operatorname{ext}_{a}(\ell)\right\} \Rightarrow$ $l s \in \operatorname{sol}\left(a_{i} \rightarrow^{*} a_{j}\right)$, up to supersets removing. One can easily show that Property 1 is verified with such a construction. The complexity of this construction is exponential in the number of local states within one automaton and polynomial in the number of automata. Alternative constructions may also provide sound (and not necessarily equal) sol.

\subsection{Graph of Local Causality}

Given a local state $a_{j} \in \mathbf{L S}$ and an initial context $\varsigma$, the reachability of $a_{i}$ is equivalent to the realization of any objective $a_{i} \rightarrow^{*} a_{j}$, with $a_{i} \in \varsigma(a)$. By definition, if $a_{j}$ is reachable from $\varsigma$, there exists $l s \in \operatorname{sol}\left(a_{i} \rightarrow^{*} a_{j}\right)$ such that, $\forall b_{k} \in l s, b_{k}$ is reachable from $\varsigma$.

The (directed) Graph of Local Causality (GLC, Def. 7) relates this recursive reasoning from a given set of local states $\omega \subseteq \mathbf{L S}$ by linking every local state $a_{j}$ to all objectives $a_{i} \rightarrow^{*} a_{j}, a_{i} \in \varsigma(a)$; every objective $P$ to its solutions $\langle P, l s\rangle \in \mathbf{S o l}$; every solution $\langle P, l s\rangle$ to its local states $b_{k} \in l s$. A GLC is said to be sound if sol is sound for all referenced objectives (Property 2).

Definition 7 (Graph of Local Causality). Given a context $\varsigma$ and a set of local states $\omega \subseteq \mathbf{L S}$, the Graph of Local Causality $(G L C) \mathcal{A}_{\varsigma}^{\omega} \triangleq\left(V_{\varsigma}^{\omega}, E_{\varsigma}^{\omega}\right)$, with $V_{\varsigma}^{\omega} \subseteq \mathbf{L S} \cup \mathbf{O b j} \cup \mathbf{S o l}$ and $E_{\varsigma}^{\omega} \subseteq V_{\varsigma}^{\omega} \times V_{\varsigma}^{\omega}$, is the smallest structure satisfying:

$$
\begin{aligned}
\omega & \subseteq V_{\varsigma}^{\omega} \\
a_{i} \in V_{\varsigma}^{\omega} \cap \mathbf{L S} & \Leftrightarrow\left\{\left(a_{i}, a_{j} \rightarrow^{*} a_{i}\right) \mid a_{j} \in \varsigma\right\} \subseteq E_{\varsigma}^{\omega} \\
a_{i} \rightarrow^{*} a_{j} \in V_{\varsigma}^{\omega} \cap \mathbf{O b j} & \Leftrightarrow\left\{\left(a_{i} \rightarrow^{*} a_{j},\left\langle a_{i} \rightarrow^{*} a_{j}, l s\right\rangle\right) \mid\left\langle a_{i} \rightarrow^{*} a_{j}, l s\right\rangle \in \mathbf{S o l}\right\} \subseteq E_{\varsigma}^{\omega} \\
\langle P, l s\rangle \in V_{\varsigma}^{\omega} \cap \mathbf{S o l} & \Leftrightarrow\left\{\left(\langle P, l s\rangle, a_{i}\right) \mid a_{i} \in l s\right\} \subseteq E_{\varsigma}^{\omega} .
\end{aligned}
$$

Property 2 (Sound Graph of Local Causality). A GLC $\mathcal{A}_{\varsigma}^{\omega}$ is sound if, $\forall P \in$ $V_{\varsigma}^{\omega} \cap \mathbf{O b j}, \operatorname{sol}(P)$ is sound. 


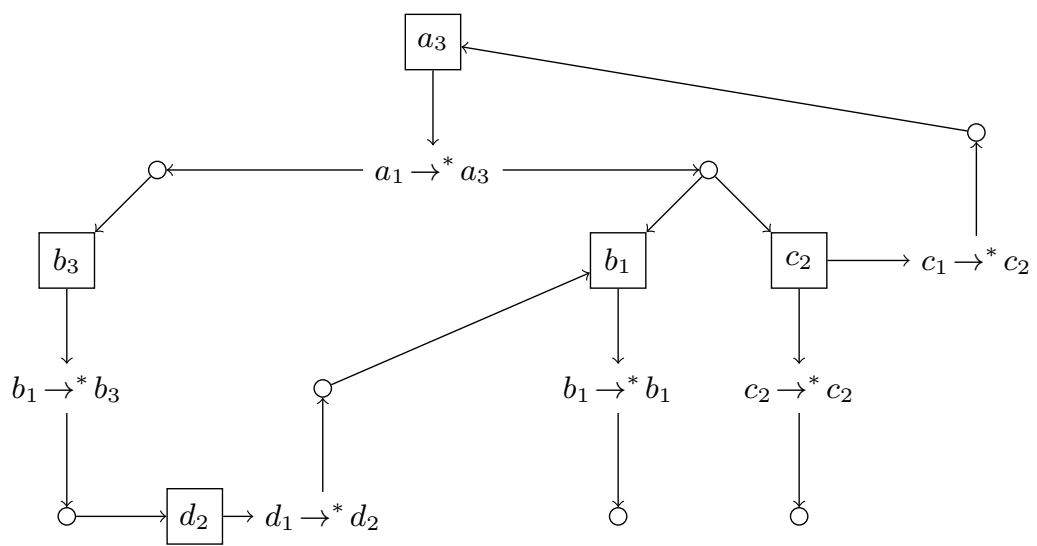

Fig. 2. Example of Graph of Local Causality that is sound for the automata network defined in Example 1

This structure can be constructed starting from local states in $\omega$ and by iteratively adding the imposed children. It is worth noticing that this graph can contain cycles. In the worst case, $\# V_{\varsigma}^{\omega}=\# \mathbf{L S}+\# \mathbf{O b j}+\# \mathbf{S o l}$ and $\# E_{\varsigma}^{\omega}=$ $\# \mathbf{O b j}+\# \mathbf{S o l}+\sum_{\langle P, l s\rangle \in \mathbf{S o l}} \# l s$.

Example 1. Fig. 2 shows an example of GLC. Local states are represented by boxed nodes and elements of Sol by small circles.

For instance, such a GLC is sound for the following automata network $(\Sigma, S, \mathcal{L}, T)$, with initial context $\varsigma=\{a \mapsto\{1\} ; b \mapsto\{1\} ; c \mapsto\{1,2\} ; d \mapsto\{2\}\}$ :

$$
\begin{aligned}
\Sigma & =\{a, b, c, d\} & \mathcal{L} & =\left\{\ell_{1}, \ell_{2}, \ell_{3}, \ell_{4}, \ell_{5}, \ell_{6}\right\} \\
S(a) & =[1 ; 3] & T(a) & =\left\{1 \stackrel{\ell_{2}}{\longrightarrow} 2 ; 2 \stackrel{\ell_{3}}{\longrightarrow} 3 ; 1 \stackrel{\ell_{1}}{\longrightarrow} 3 ; 3 \stackrel{\ell_{4}}{\longrightarrow} 2\right\} \\
S(b) & =[1 ; 3] & T(b) & =\left\{1 \stackrel{\ell_{2}}{\longrightarrow} 2 ; 1 \stackrel{\ell_{5}}{\longrightarrow} 3 ; 1 \stackrel{\ell_{6}}{\longrightarrow} 1 ; 3 \stackrel{\ell_{1}}{\longrightarrow} 2\right\} \\
S(c) & =[1 ; 2] & T(c) & =\left\{1 \stackrel{\ell_{4}}{\longrightarrow} 2 ; 2 \stackrel{\ell_{3}}{\longrightarrow} 1\right\} \\
S(d) & =[1 ; 2] & T(d) & =\left\{1 \stackrel{\ell_{6}}{\longrightarrow} 2 ; 2 \stackrel{\ell_{5}}{\longrightarrow} 1\right\}
\end{aligned}
$$

For example, within automata $a$, there are two acyclic sequences from 1 to 3 : $1 \stackrel{\ell_{2}}{\longrightarrow} 2 \stackrel{\ell_{3}}{\longrightarrow} 3$ and $1 \stackrel{\ell_{1}}{\longrightarrow} 3$. Hence, if $a_{3}$ is reached from $a_{1}$, then necessarily, one of these two sequences has to be used (but not necessarily consecutively). For each of these transitions, the transition label is shared by exactly one local state in another automaton: $b_{1}, c_{2}, b_{3}$ for $\ell_{2}, \ell_{3}, \ell_{1}$, respectively. Therefore, if $a_{3}$ is reached from $a_{1}$, then necessarily either both $b_{1}$ and $c_{2}$, or $b_{3}$ have been reached before. Hence sol $\left(a_{1} \rightarrow^{*} a_{3}\right)=\left\{\left\{b_{1}, c_{2}\right\},\left\{b_{3}\right\}\right\}$ is sound, as disabling either $b_{1}$ and $b_{3}$, or $c_{2}$ and $b_{3}$, would remove any possibility to reach $a_{3}$ from $a_{1}$. 


\section{$3 \quad$ Necessary Local States for Reachability}

We assume a global sound GLC $\mathcal{A}_{\varsigma}^{\omega}=\left(V_{\varsigma}^{\omega}, E_{\varsigma}^{\omega}\right)$, with the usual accessors for the direct relations of nodes:

$$
\begin{array}{cr}
\text { children : } V_{\varsigma}^{\omega} \mapsto \wp\left(V_{\varsigma}^{\omega}\right) & \text { parents : } V_{\varsigma}^{\omega} \mapsto \wp\left(V_{\varsigma}^{\omega}\right) \\
\text { children }(n) \triangleq\left\{m \in V_{\varsigma}^{\omega} \mid(n, m) \in E_{\varsigma}^{\omega}\right\} & \text { parents }(n) \triangleq\left\{m \in V_{\varsigma}^{\omega} \mid(m, n) \in E_{\varsigma}^{\omega}\right\}
\end{array}
$$

Given a set of local states $\mathcal{O} b s \subseteq \mathbf{L S}$, this section introduces an algorithm computing upon $\mathcal{A}_{\varsigma}^{\omega}$ the set $\mathbb{V}\left(a_{i}\right)$ of minimal cut $N$-sets of local states in $\mathcal{O} b s$ that are necessary for the independent reachability of each local state $a_{i} \in$ $\mathbf{L S} \cap V_{\varsigma}^{\omega}$. The minimality criterion actually states that $\forall l s \in \mathbb{V}\left(a_{i}\right)$, there is no different $l s^{\prime} \in \mathbb{V}\left(a_{i}\right)$ such that $l s^{\prime} \subset l s$.

Assuming a first valuation $\mathbb{V}$ (Def. 8) associating to each node its cut $N$-sets, the cut $N$-sets for the node $n$ can be refined using update $(\mathbb{V}, n$ ) (Def. 9):

- if $n$ is a solution $\langle P, l s\rangle \in \mathbf{S o l}$, it is sufficient to prevent the reachability of any local state in $l s$ to cut $n$; therefore, the cut $N$-sets results from the union of the cut $N$-sets of $n$ children (all local states).

- If $n$ is an objective $P \in \mathbf{O b j}$, all its solutions (in sol $(P)$ ) have to be cut in order to ensure that $P$ is not realizable: hence, the cut $N$-sets result from the product of children cut $N$-sets (all solutions).

- If $n$ is a local state $a_{i}$, it is sufficient to cut all its children (all objectives) to prevent the reachability of $a_{i}$ from any state in the context $\varsigma$. In addition, if $a_{i} \in \mathcal{O} b s,\left\{a_{i}\right\}$ is added to the set of its cut $N$-sets.

Definition 8 (Valuation $\mathbb{V})$. A valuation $\mathbb{V}: V_{\varsigma}^{\omega} \mapsto \wp(\wp \leq N(\mathcal{O} b s))$ is a mapping from each node of $\mathcal{A}_{\varsigma}^{\omega}$ to a set of $N$-sets of local states. Val is the set of all valuations. $\mathbb{V}_{0} \in \mathbf{V a l}$ refers to the valuation such that $\forall n \in V_{\varsigma}^{\omega}, \mathbb{V}_{0}(n)=\emptyset$.

Definition 9 (update : Val $\times V_{\varsigma}^{\omega} \mapsto$ Val).

$$
\text { update }(\mathbb{V}, n) \triangleq \begin{cases}\mathbb{V}\left\{n \mapsto \zeta^{N}\left(\bigcup_{m \in \operatorname{children}(n)} \mathbb{V}(m)\right)\right\} & \text { if } n \in \mathbf{\text { Sol }} \\ \mathbb{V}\left\{n \mapsto \zeta^{N}\left(\widetilde{\prod}_{m \in \operatorname{children}(n)} \mathbb{V}(m)\right)\right\} & \text { if } n \in \mathbf{O b j} \\ \mathbb{V}\left\{n \mapsto \zeta^{N}\left(\widetilde{\prod}_{m \in \operatorname{children}(n)} \mathbb{V}(m)\right)\right\} & \text { if } n \in \mathbf{L S} \backslash \mathcal{O} b s \\ \mathbb{V}\left\{n \mapsto \zeta^{N}\left(\left\{\left\{a_{i}\right\}\right\} \cup \widetilde{\prod}_{m \in \operatorname{children}(n)} \mathbb{V}(m)\right)\right\} & \text { if } n \in \mathbf{L S} \cap \mathcal{O} b s\end{cases}
$$

where $\zeta^{N}\left(\left\{e_{1}, \ldots, e_{n}\right\}\right) \triangleq\left\{e_{i} \mid i \in[1 ; n] \wedge \# e_{i} \leq N \wedge \nexists j \in[1 ; n], j \neq i, e_{j} \subset e_{i}\right\}$, $e_{i}$ being sets, $\forall i \in[1 ; n]$.

Starting with $\mathbb{V}_{0}$, one can repeatedly apply update on each node of $\mathcal{A}_{\varsigma}^{\omega}$ to refine its valuation. Only nodes where one of their children value has been modified should be considered for updating.

Hence, the order of nodes updates should follow the topological order of the GLC, where children have a lower rank than their parents (i.e., children are treated before their parents). If the graph is actually acyclic, then it is sufficient 


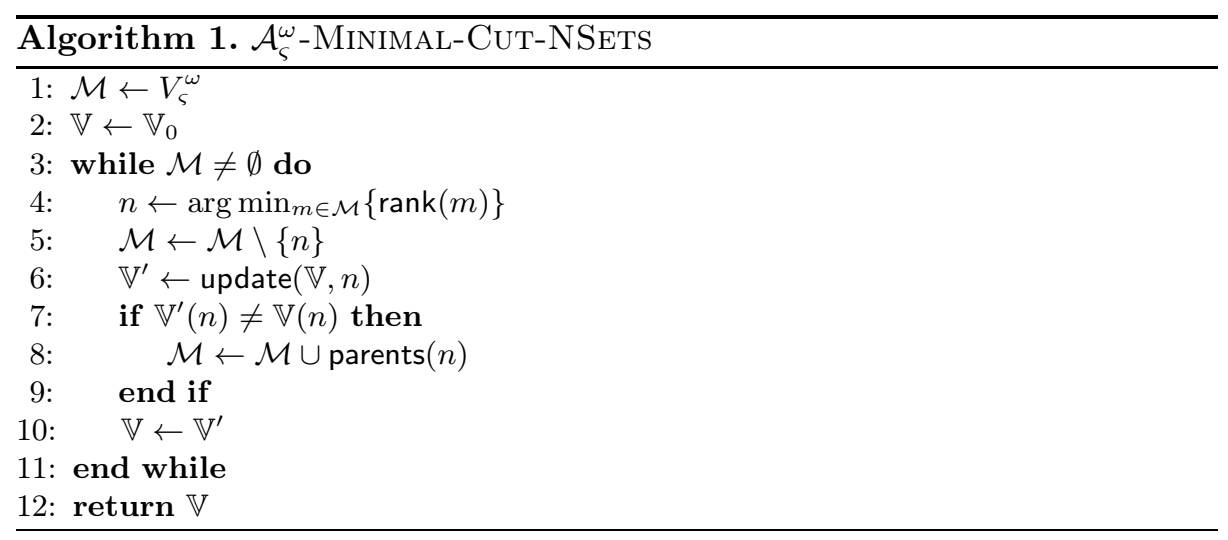

to update the value of each node only once. In the general case, i.e. in the presence of Strongly Connected Components (SCCs) - nodes belonging to the same SCC have the same rank - , the nodes within a SCC have to be iteratively updated until the convergence of their valuation.

Algorithm 1 formalizes this procedure where $\operatorname{rank}(n)$ refers to the topological rank of $n$, as it can be derived from Tarjan's strongly connected components algorithm [23], for example. The node $n \in V_{\varsigma}^{\omega}$ to be updated is selected as being the one having the least rank amongst the nodes to update (delimited by $\mathcal{M}$ ). In the case where several nodes with the same lowest rank are in $\mathcal{M}$, they can be either arbitrarily or randomly picked. Once picked, the value of $n$ is updated. If the new valuation of $n$ is different from the previous, the parents of $n$ are added to the list of nodes to update (lines 6-8 in Algorithm 1).

Lemma 1 states the convergence of Algorithm1 and Theorem 1 its correctness: for each local state $a_{i} \in V_{\varsigma}^{\omega} \cap \mathbf{L S}$, each set of local states $k l s \in \mathbb{V}\left(a_{i}\right)$ (except $\left\{a_{i}\right\}$ singleton) references the local states that are all necessary to reach prior to the reachability of $a_{i}$ from any state in $\varsigma$. Hence, if all the local states in $k l s$ are disabled in $\mathcal{S} y s, a_{i}$ is not reachable from any state in $\varsigma$.

Lemma 1. $\mathcal{A}_{\varsigma}^{\omega}$-MinimaL-CUT-NSETS always terminates.

Proof. Remarking that $\wp(\wp \leq N(\mathcal{O} b s))$ is finite, defining a partial ordering such that $\forall v, v^{\prime} \in \wp(\wp \leq N(\mathcal{O} b s)), v \succeq v^{\prime} \Leftrightarrow \zeta^{N}(v)=\zeta^{N}\left(v \cup v^{\prime}\right)$, and noting $\mathbb{V}^{k} \in$ Val the valuation after $k$ iterations of the algorithm, it is sufficient to prove that $\mathbb{V}^{k+1}(n) \succeq \mathbb{V}^{k}(n)$. Let us define $v_{1}, v_{2}, v_{1}^{\prime}, v_{2}^{\prime} \in \wp(\wp \leq N(\mathcal{O} b s))$ such that $v_{1} \succeq v_{1}^{\prime}$ and $v_{2} \succeq v_{2}^{\prime}$. We can easily check that $v_{1} \cup v_{2} \succeq v_{1}^{\prime} \cup v_{2}^{\prime}$ (hence proving the case when $n \in \mathbf{S o l})$. As $\zeta^{N}\left(v_{1}\right)=\zeta^{N}\left(v_{1} \cup v_{1}^{\prime}\right) \Leftrightarrow \forall e_{1}^{\prime} \in v_{1}^{\prime}, \exists e_{1} \in v_{1}: e_{1} \subseteq e_{1}^{\prime}$, we obtain that $\forall\left(e_{1}^{\prime}, e_{2}^{\prime}\right) \in v_{1}^{\prime} \times v_{2}^{\prime}, \exists\left(e_{1}, e_{2}\right) \in v_{1} \times v_{2}: e_{1} \subseteq e_{1}^{\prime} \wedge e_{2} \subseteq e_{2}^{\prime}$. Hence $e_{1} \cup e_{2} \subseteq e_{1}^{\prime} \cup e_{2}^{\prime}$, therefore $\zeta^{N}\left(v_{1} \tilde{\times} v_{2} \cup v_{1}^{\prime} \tilde{\times} v_{2}^{\prime}\right)=\zeta^{N}\left(v_{1} \tilde{\times} v_{2}\right)$, i.e. $v_{1} \tilde{\times} v_{2} \succeq v_{1}^{\prime} \tilde{\times} v_{2}^{\prime}$; which proves the cases when $n \in \mathbf{O b j} \cup \mathbf{L S}$.

Theorem 1. Given a $G L C \mathcal{A}_{\varsigma}^{\omega}=\left(V_{\varsigma}^{\omega}, E_{\varsigma}^{\omega}\right)$ which is sound for the automata network $\mathcal{S} y s$, the valuation $\mathbb{V}$ computed by $\mathcal{A}_{\varsigma}^{\omega}$-MINIMAL-CUT-NSETS verifies: $\forall a_{i} \in \mathbf{L S} \cap V_{\varsigma}^{\omega}, \forall k l s \in \mathbb{V}\left(a_{i}\right) \backslash\left\{\left\{a_{i}\right\}\right\}, a_{j}$ is not reachable from $\varsigma$ within $\mathcal{S} y s \ominus k l s$. 
Table 1. Result of the execution of Algorithm 1 on the GLC in Fig. 2

\begin{tabular}{|c|c|l|}
\hline Node & rank & $\mathbb{V}$ \\
\hline$\left\langle b_{1} \rightarrow^{*} b_{1}, \emptyset\right\rangle$ & 1 & $\emptyset$ \\
$b_{1} \rightarrow^{*} b_{1}$ & 2 & $\emptyset$ \\
$b_{1}$ & 3 & $\left\{\left\{b_{1}\right\}\right\}$ \\
$\left\langle d_{1} \rightarrow^{*} d_{2},\left\{b_{1}\right\}\right\rangle$ & 4 & $\left\{\left\{b_{1}\right\}\right\}$ \\
$d_{1} \rightarrow^{*} d_{2}$ & 5 & $\left\{\left\{b_{1}\right\}\right\}$ \\
$d_{2}$ & 6 & $\left\{\left\{b_{1}\right\},\left\{d_{2}\right\}\right\}$ \\
$\left\langle b_{1} \rightarrow^{*} b_{3},\left\{d_{2}\right\}\right\rangle$ & 7 & $\left\{\left\{b_{1}\right\},\left\{d_{2}\right\}\right\}$ \\
$b_{1} \rightarrow^{*} b_{3}$ & 8 & $\left\{\left\{b_{1}\right\},\left\{d_{2}\right\}\right\}$ \\
$b_{3}$ & 9 & $\left\{\left\{b_{1}\right\},\left\{b_{3}\right\},\left\{d_{2}\right\}\right\}$ \\
$\left\langle a_{1} \rightarrow^{*} a_{3},\left\{b_{3}\right\}\right\rangle$ & 10 & $\left\{\left\{b_{1}\right\},\left\{b_{3}\right\},\left\{d_{2}\right\}\right\}$ \\
$\left\langle c_{2} \rightarrow^{*} c_{2}, \emptyset\right\rangle$ & 11 & $\emptyset$ \\
$c_{2} \rightarrow^{*} c_{2}$ & 12 & $\emptyset$ \\
$c_{2}$ & 13 & $\left\{\left\{c_{2}\right\}\right\}$ \\
$\left\langle a_{1} \rightarrow{ }^{*} a_{3},\left\{b_{1}, c_{2}\right\}\right\rangle$ & 13 & $\left\{\left\{b_{1}\right\},\left\{c_{2}\right\}\right\}$ \\
$a_{1} \rightarrow^{*} a_{3}$ & 13 & $\left\{\left\{b_{1}\right\},\left\{b_{3}, c_{2}\right\},\left\{c_{2}, d_{2}\right\}\right\}$ \\
$a_{3}$ & 13 & $\left\{\left\{a_{3}\right\},\left\{b_{1}\right\},\left\{b_{3}, c_{2}\right\},\left\{c_{2}, d_{2}\right\}\right\}$ \\
$\left\langle c_{1} \rightarrow{ }^{*} c_{2},\left\{a_{3}\right\}\right\rangle$ & 13 & $\left\{\left\{a_{3}\right\},\left\{b_{1}\right\},\left\{b_{3}, c_{2}\right\},\left\{c_{2}, d_{2}\right\}\right\}$ \\
\hline
\end{tabular}

Proof. By recurrence on the valuations $\mathbb{V}$ : the above property is true at each iteration of the algorithm.

Example 2. Table1 details the result of the execution of Algorithm1 on the GLC defined in Fig. 2. Nodes receive a topological rank, identical ranks implying the belonging to the same SCC. The (arbitrary) scheduling of the updates of nodes within a SCC follows the order in the table. In this particular case, nodes are all visited once, as $\mathbb{V}\left(\left\langle c_{2} \rightarrow^{*} c_{2}, \emptyset\right\rangle\right) \tilde{\times} \mathbb{V}\left(\left\langle c_{1} \rightarrow^{*} c_{2},\left\{a_{3}\right\}\right\rangle\right)=\emptyset$ (hence update $\left(\mathbb{V}, c_{2}\right)$ does not change the valuation of $\left.c_{2}\right)$. Note that in general, several iterations of update may be required to reach a fixed point.

It is worth noticing that the GLC abstracts several dynamical constraints in the underlying automata networks, such as the ordering of transitions, or the synchronous updates of the global state. In that sense, GLC over-approximates the dynamics of the network, and the resulting cut sets are under-approximating the complete cut sets of the concrete model: any computed cut sets is a superset of a complete cut set (potentially equal).

\section{Application to Systems Biology}

Automata networks, as presented in Def. 1, subsume Boolean and discrete networks, synchronous and asynchronous, that are widely used for the qualitative modelling of dynamics of biological networks 9 24/17/2 $7 / 186]$.

A cut set, as extracted by our algorithm, informs that at least one of the component in the cut set has to be present in the specified local state in order to achieve the wanted reachability. A local state can represent, for instance, 
an active transcription factor or the absence of a certain protein. It provides potential therapeutic targets if the studied reachability is involved in a disease by preventing all the local states of a cut set to act, for instance using gene knock-out or knock-in techniques.

We first discuss and compare our methodology with the intervention sets analysis within biological models developed by S. Klamt et al., and provide some benchmarks on a few examples.

Thanks to the use of the intermediate GLC and to the absence of candidate enumeration, our new method makes tractable the cut sets analysis on very large models. We present a recent application of our results to the analysis of a very large scale Boolean model of biological pathway interactions involving 9000 components. To our knowledge, this is the first attempt of a formal dynamical analysis on such a large scale model.

\subsection{Related Work}

The general related work having been discussed in Sect. 1] we deepen here the comparison of our method with the closest related work: the analysis of Intervention Sets (ISs) [19]. Cut sets and ISs have a reversed logic: an IS specifies local states to enforce in order to ensure a particular behaviour to occur; a cut set specifies local states to disable in order to prevent a particular behaviour to occur. In the scope of Boolean models of signalling networks, ISs are computed for the reachability of a given fixed point (steady state) which can be partially defined. Their method is complete: all minimal ISs are computed.

Nevertheless, the semantics and the computation of ISs have some key differences with our computed cut sets. First, they focus only on the reachability of (logical) steady states, which is a stronger condition than the transient reachability that we are considering. Then, the steady states are computed using a three-valued logic which allows to cope with undefined (initial) local states, but which is different from the notion of context that we use in this paper for specifying the initial condition.

Such differences make difficult a proper comparison of inferred cut sets. We can however expect that any cut sets found by our method has a corresponding IS in the scope of Boolean networks with a single initial state.

To give a practical insight on the relation between the two methods, we compare the results for two signalling networks, both between a model specified with CellNetAnalyser [11] to compute ISs and a model specified in the Process Hitting framework, a particular restriction of asynchronous automata networks [15], to compute our cut sets. Process Hitting models have been built in order to over-approximate the dynamics considered for the computation of IS: 1 .

Tcell. Applied to a model of the T-cell receptor signalling between 40 components [12, we are interested in preventing the activation of the transcription factor $A P 1$. For an instance of initial conditions, and limiting the computations to 3 -sets, 31 ISs have been identified (28 1-sets, 32 -sets, 0 3-set), whereas our

\footnotetext{
${ }^{1}$ Models and scripts available at http://loicpauleve.name/cutsets.tbz2
} 
algorithm found 29 cut sets (21 1-sets and 8 2-sets), which are all matching an IS (23 are identical, 6 strictly including ISs). ISs are computed in 0.69 s while our algorithm under-approximates the cut sets in $0.006 \mathrm{~s}$. Different initial states give comparable results.

Egfr. Applied to a model of the epidermal growth factor receptor signalling pathway of 104 components [18], we are interested in preventing the activation of the transcription factor $A P 1$. For an instance of initial conditions, and limiting the computations to 3 -sets, 25 ISs have been identified (19 1-sets, 3 2-sets, 33 sets), whereas our algorithm found 14 cut sets (14 1-sets), which are all included in the ISs. ISs are computed in 98s while our algorithm under-approximates the cut sets in 0.004s. Different initial states give comparable results.

As expected with the different semantics of models and cut sets, resulting ISs matches all the cut sets identified by our algorithm, and provides substantially more sets. The execution time is much higher for ISs as they rely on candidate enumeration in order to provide complete results, whereas our method was designed to prevent such an enumeration but under-approximates the cut sets.

In order to appreciate the under-approximation done by our method at a same level of abstraction and with identical semantics, we compare the cut sets identified by our algorithm with the cut sets obtained using a naive, but complete, computation. The naive computation enumerates all cut set candidates and, for each of them, disable the local states in the model and perform model-checking to verify if the target local state is still reachable. In the particular case of these two models, and limiting the cut sets to 3 and 2-sets respectively for the sake of tractability, no additional cut set has been uncovered by the complete method. Such a good under-approximation could be partially explained by the restrictions imposed on the causality by the Process Hitting framework, making the GLC a tight over-approximation of the dynamics [16].

\subsection{Very Large Scale Application to Pathway Interactions}

In order to support the scalability of our approach, we apply the proposed algorithm to a very large model of biological interactions, actually extracted from the PID database [20] referencing various influences (complex formation, inductions (activations) and inhibitions, transcriptional regulation, etc.) between more than 9000 biological components (proteins, genes, ions, etc.).

Amongst the numerous biological components, the activation of some of them are known to control key mechanisms of the cell dynamics. Those activations are the consequence of intertwining signalling pathways and depend on the environment of the cell (represented by the presence of certain entry-point molecules). Uncovering the environmental and intermediate components playing a major role in these signalling dynamics is of great biological interest.

The full PID database has been interpreted into the Process Hitting framework, a subclass of asynchronous automata networks, from which the derivation of the GLC has been addressed in previous work [16]. The obtained model gathers components representing either biological entities modelled as boolean value (absent or present), or logical complexes. When a biological component has several 
competing regulators, the precise cooperations are not detailed in the database, so we use of two different interpretations: all (resp. one of) the activators and none (resp. all but one of) the inhibitors have to be present in order to make the target component present. This leads to two different discrete models of PID that we refer to as whole_PID_AND and whole_PID_OR, respectively.

Focusing on whole_PID_OR, the Process Hitting model relates more than 21000 components, either biological or logical, containing between 2 and 4 local states. Such a system could actually generate $2^{33874}$ states. 3136 components act as environment specification, which in our boolean interpretation leads to $2^{3136}$ possible initial states, assuming all other components start in the absent state.

We focus on the (independent) reachability of active SNAIL transcription factor, involved in the epithelial to mesenchymal transition [14, and of active p15INK4b and p21CIP1 cyclin-dependent kinase inhibitors involved in cell cycle regulation [3. The GLC relates 20045 nodes, including 5671 component local states (biological or logical); it contains 6 SCCs with at least 2 nodes, the largest being composed of 10238 nodes and the others between 20 and 150 .

Table 2 shows the results of a prototype implementation 2 of Algorithm 1 for the search of up to the 6 -sets of biological component local states. One can observe that the execution time grows very rapidly with $N$ compared to the number of visited nodes. This can be explained by intermediate nodes having a large set of cut $N$-sets leading to a costly computation of products.

While the precise biological interpretation of identified $N$-sets is out of the scope of this paper, we remark that the order of magnitude of the number of cut sets can be very different (more than 1000 cut 6-sets for SNAIL; none cut 6-sets for p21CIP1, except the gene that produces this protein). It supports a notion of robustness for the reachability of components, where the less cut sets, the more robust the reachability to various perturbations.

Applied to the whole_PID_AND model, our algorithm find in general much more cut $N$-sets, due to the conjunctive interpretation. This brings a significant increase in the execution time: the search up to the cut 5 -sets took $1 \mathrm{~h}$, and the 6 -sets leads to an out-of-memory failure.

Table 2. Results for the computation of cut $N$-sets for 3 local states. For each $\mathrm{N}$, only the number of additional N-sets is displayed.

\begin{tabular}{|c|c|c|c|c|c|c|}
\hline \multirow{2}{*}{ Model } & \multirow{2}{*}{$\mathrm{N}$} & \multirow{2}{*}{ Visited nodes } & \multirow{2}{*}{ Exec. time } & \multicolumn{3}{|c|}{ Nb. of resulting N-sets } \\
\hline & & & & SNAIL $_{1}$ & p15INK4b & $\mathrm{p} 21 \mathrm{CIP} 1_{1}$ \\
\hline \multirow{6}{*}{ whole_PID_OR } & 1 & 29022 & $0.9 \mathrm{~s}$ & 1 & 1 & 1 \\
\hline & 2 & 36602 & $1.6 \mathrm{~s}$ & $\overline{+6}$ & +6 & +0 \\
\hline & 3 & 44174 & $5.4 \mathrm{~s}$ & +0 & +92 & +0 \\
\hline & 4 & 54322 & $39 \mathrm{~s}$ & +30 & +60 & +0 \\
\hline & 5 & 68214 & $8.3 \mathrm{~m}$ & +90 & +80 & $\overline{+0}$ \\
\hline & 6 & 90902 & $2.6 \mathrm{~h}$ & +930 & +208 & +0 \\
\hline
\end{tabular}

${ }^{2}$ Implemented as part of the PINT software - http://process.hitting.free.fr Models and scripts available at http://loicpauleve.name/cutsets.tbz2 
The very large number of involved components makes intractable the naive exact algorithm consisting in enumerating all possible $N$-sets candidates and verifying the concerned reachability using model-checking. Similarly, making such a model fit into other frameworks, such as CellNetAnalyser (see previous subsection) is a challenging task, and might be considered as future work.

\section{Discussion}

We presented a new method to efficiently compute cut sets for the reachability of a local state of a component within networks of finite automata from any state delimited by a provided so-called context. Those cut sets are sets of automata local states such that disabling the activity of all local states of a cut set guarantees to prevent the reachability of the concerned local state. Automata networks are commonly used to represent the qualitative dynamics of interacting biological systems, such as signalling networks. The computation of cut sets can then lead to propose potential therapeutic targets that have been formally identified from the model for preventing the activation of a particular molecule.

The proposed algorithm works by propagating and combining the cut sets of local states along a Graph of Local Causality (GLC), that we introduce here upon automata networks. A GLC relates the local states that are necessary to occur prior to the reachability of the concerned local state. Several constructions of a GLC are generally possible and depend on the semantics of the model. We gave an example of such a construction for automata networks. That GLC has a size polynomial in the total number of local states in the network, and exponential in the number of local states within one automaton. Note that the core algorithm for computing the cut sets only requires as input a GLC satisfying a soundness property that can be easily extended to discrete systems that are more general than the automata networks considered here.

The computed cut sets are an under-approximation of the complete cut sets as the GLC abstracts several dynamical constraints from the underlying concrete model: any computed cut sets is a superset of a concrete cut set (potentially equal), some can be missed. Our algorithm prevents a costly enumeration of the potential sets of candidates, and aims at being tractable on very large networks.

A prototype implementation of our algorithm has been successfully applied to the extraction of cut sets from a Boolean model of a biological system involving more than 9000 interacting components. To our knowledge this is the first attempt of such a dynamical analysis for such large biological models. We note that most of the computation time is due to products between large sets of cut $N$-sets. To partially address this issue, we use of prefix trees to represent set of sets on which we have specialized operations to stick to sets of $N$-sets (Appendix $\mathrm{A}^{3}$ ). There is still room for improvement as our prototype does not implement any caching or variable re-ordering.

The work presented in this paper can be extended in several ways, notably with a posterior enlarging of the cut sets. Because the algorithm computes the

\footnotetext{
${ }^{3}$ Available at http://loicpauleve.name/CAV2013-SI.pdf
} 
cut $N$-sets for each node in the GLC, it is possible to construct a posteriori cut sets with a greater cardinality by chaining them. For instance, let $k p s \in \mathbb{V}\left(a_{i}\right)$ be a cut $N$-set for the reachability of $a_{i}$, for each $b_{j} \in k p s$ and $k p s^{\prime} \in \mathbb{V}\left(b_{j}\right)$, $\left(k p s \backslash\left\{b_{j}\right\}\right) \cup k p s^{\prime}$ is a cut set for $a_{i}$. In our biological case study, this method could be recursively applied until cut sets are composed of states of automata only acting for the environmental input.

With respect to the defined computation of cut $N$-sets, one could also derive static reductions of the GLC. Indeed, some particular nodes and arcs of the GLC can be removed without affecting the final valuation of nodes. A simple example are nodes representing objectives having no solution: such nodes can be safely removed as they bring no candidate $N$-sets for parents processes. These reductions conduct to both speed-up of the proposed algorithm but also to more compact representations for the reachability causality.

In addition of providing potential targets to prevent the occurrence of some behaviours, it may be crucial to ensure that the modified system keep satisfying important dynamical properties, as it is tackled with constrained minimal cut sets [48. Currently, such constraints could be verified a posteriori in order to filter the computed cut sets that break important dynamics requirements. Taking advantage of those constraints during the computation is hence an promising research direction for large-scale applications.

Acknowledgements. LP and GA acknowledge the partial support of the French National Agency for Research (ANR-10-BLANC-0218 BioTempo project). The work of LP was supported by the Swiss SystemsX.ch project. The work of HK was supported by the Swiss National Science Foundation, grant no. PP00P2 128503.

\section{References}

1. Bernardinello, L., De Cindio, F.: A survey of basic net models and modular net classes. In: Rozenberg, G. (ed.) APN 1992. LNCS, vol. 609, pp. 304-351. Springer, Heidelberg (1992)

2. Bernot, G., Cassez, F., Comet, J.P., Delaplace, F., Müller, C., Roux, O.: Semantics of biological regulatory networks. Electronic Notes in Theoretical Computer Science 180(3), 3-14 (2007)

3. Drabsch, Y., Ten Dijke, P.: TGF- $\beta$ signalling and its role in cancer progression and metastasis. Cancer Metastasis Rev. 31(3-4), 553-568 (2012)

4. Hädicke, O., Klamt, S.: Computing complex metabolic intervention strategies using constrained minimal cut sets. Metabolic Engineering 13(2), 204-213 (2011)

5. Harel, D., Kupferman, O., Vardi, M.Y.: On the complexity of verifying concurrent transition systems. Information and Computation 173(2), 143-161 (2002)

6. Hinkelmann, F., Laubenbacher, R.: Boolean models of bistable biological systems. Discrete and Continuous Dynamical Systems - Series S 4(6), 1443-1456 (2011)

7. de Jong, H.: Modeling and simulation of genetic regulatory systems: A literature review. Journal of Computational Biology 9, 67-103 (2002)

8. Jungreuthmayer, C., Zanghellini, J.: Designing optimal cell factories: integer programming couples elementary mode analysis with regulation. BMC Systems Biology 6(1), 103 (2012) 
9. Kauffman, S.A.: Metabolic stability and epigenesis in randomly connected nets. Journal of Theoretical Biology 22, 437-467 (1969)

10. Klamt, S., Gilles, E.D.: Minimal cut sets in biochemical reaction networks. Bioinformatics 20(2), 226-234 (2004)

11. Klamt, S., Saez-Rodriguez, J., Gilles, E.: Structural and functional analysis of cellular networks with cellnetanalyzer. BMC Systems Biology 1(1), 2 (2007)

12. Klamt, S., Saez-Rodriguez, J., Lindquist, J., Simeoni, L., Gilles, E.: A methodology for the structural and functional analysis of signaling and regulatory networks. BMC Bioinformatics 7(1) 7(1), 56 (2006)

13. Lee, W.S., Grosh, D.L., Tillman, F.A., Lie, C.H.: Fault tree analysis, methods, and applications - a review. IEEE Transactions on Reliability R-34, 194-203 (1985)

14. Moustakas, A., Heldin, C.H.: Signaling networks guiding epithelial-mesenchymal transitions during embryogenesis and cancer progression. Cancer Sci. 98(10), 15121520 (2007)

15. Paulevé, L., Magnin, M., Roux, O.: Refining dynamics of gene regulatory networks in a stochastic $\pi$-calculus framework. In: Priami, C., Back, R.-J., Petre, I., de Vink, E. (eds.) Transactions on Computational Systems Biology XIII. LNCS, vol. 6575, pp. 171-191. Springer, Heidelberg (2011)

16. Paulevé, L., Magnin, M., Roux, O.: Static analysis of biological regulatory networks dynamics using abstract interpretation. Mathematical Structures in Computer Science 22(4), 651-685 (2012)

17. Richard, A.: Negative circuits and sustained oscillations in asynchronous automata networks. Advances in Applied Mathematics 44(4), 378-392 (2010)

18. Samaga, R., Saez-Rodriguez, J., Alexopoulos, L.G., Sorger, P.K., Klamt, S.: The logic of egfr/erbb signaling: Theoretical properties and analysis of high-throughput data. PLoS Comput. Biol. 5(8), e1000438 (2009)

19. Samaga, R., Von Kamp, A., Klamt, S.: Computing combinatorial intervention strategies and failure modes in signaling networks. Journal of Computational Biology 17(1), 39-53 (2010)

20. Schaefer, C.F., Anthony, K., Krupa, S., Buchoff, J., Day, M., Hannay, T., Buetow, K.H.: PID: The Pathway Interaction Database. Nucleic Acids Res. 9, D674-D679 (2009)

21. Shier, D.R., Whited, D.E.: Iterative algorithms for generating minimal cutsets in directed graphs. Networks 16(2), 133-147 (1986)

22. Tang, Z., Dugan, J.: Minimal cut set/sequence generation for dynamic fault trees. In: Reliability and Maintainability, 2004 Annual Symposium, RAMS (2004)

23. Tarjan, R.: Depth-first search and linear graph algorithms. SIAM Journal on Computing 1(2), 146-160 (1972)

24. Thomas, R.: Boolean formalization of genetic control circuits. Journal of Theoretical Biology 42(3), 563-585 (1973) 